\title{
Silicon-based Photonic Ring Resonators for Optical OFDM Demultiplexing
}

\author{
R. Vilar, F. Ramos, J. Marti \\ Valencia Nanophotonics Technology Center, Universidad Politécnica de Valencia 46022, Valencia, Spain. \\ E-mail: rutvima@ntc.upv.es
}

\begin{abstract}
We propose a compact integrated OOFDM demultiplexer based on silicon-based photonic ring resonators, providing cost-effective solution and low energy consumption. 160-Gb/s OOFDM demultiplexing operation is validated, showing excellent BER performance with error-free operation.
\end{abstract}

\section{Introduction}

Silicon photonics is a technology platform that enables high density photonic integrated circuits, allows high-bandwidth data processing, and offers low manufacturing cost given by the high compatibility with the already established micro-electronics industry. These features make silicon-based photonic devices a promising candidate to carry out signal processing tasks in future high capacity communication systems.

Optical orthogonal frequency division multiplexing (OOFDM) is receiving much attention as a potential technology for increasing transport capacities beyond $100 \mathrm{~Gb} / \mathrm{s}$ due to its high spectral efficiency and advanced robustness against chromatic dispersion and polarization mode dispersion [1]. The concept underlying OOFDM is the generation of an analog symbol signal (OFDM channel) whose spectral components include multiple subcarriers modulated in parallel by independent data streams with relatively low rates. All the subcarriers are orthogonal to each other so that their spectra overlap, which makes subchannel extraction by means of conventional optical bandpass filtering impossible. As a result, conventional OFDM signal reception is based on discrete fast Fourier transform (FFT) processing [2]. To deal with bit rates beyond $100 \mathrm{~Gb} / \mathrm{s}$, the FFT must be done at the optical domain, thus allowing the electrical speed requirements to be relaxed and the energy consumption reduced. In the literature, some all-optical FFT implementations have been proposed [3-4]. Such all-optical FFT circuits can be applied to demultiplex the optical OFDM channel and are usually comprised of several stages, each of which contains appropriate temporal delays and phase shifts. Following this concept, the aim of this work is to exploit the advantages of silicon-based photonic ring resonators (RR) for designing a compact integrated OOFDM demultiplexer. The principle of operation of such a device based on silicon ring resonators is explained and successful simultaneous demultiplexing of four OFDM subchannels consisting of 40-Gb/s-per subchannel NRZ-OOK signals is demonstrated. Simulation results show excellent BER performance for each demultiplexed OFDM subchannel and error free operation is obtained.

\section{Integrated Photonic Ring Resonators for OOFDM demultiplexing}

In this section, we propose a fully all-optical OFDM demultiplexing scheme which is based on the cascade of several ring resonators using an architecture similar to that presented in [4] for optical FFT implementation.

For OFDM applications, the FFT of order $N$ is able to discriminate $N$ individual frequency components of the input signal. Indeed, the FFT acts as a periodic filter in the frequency domain with a Free Spectral Range (FSR) of $N \Delta \omega$ (where $\Delta \omega$ is the subcarrier frequency spacing). Hence, the FFT of order $N\left(N=2^{p}, \mathrm{p}\right.$ : integer) can be implemented optically by cascading a $N / 2$-th order FFT and a delay interferometer (DI) with delay $T / N$ and a specific phase shift. Each DI is also a periodic filter with FSR equal to $N \Delta \omega / 2 p$ where $p$ is the index of the FFT stage. Thus, by cascading a sufficient number of DIs with correct delay and phase, any arbitrary frequency component can be isolated. Following this idea, we propose a scheme in which each DI is replaced by a ring resonator based filter. Indeed, a ring resonator is a wavelength-selective device which shows resonances for specific wavelengths and it can be used to implement a wide variety of filters with a periodic response determined by the FSR. Then, the optical circuit for $N$-subcarrier demultiplexing $\left(N=2^{p}\right)$ consists of a cascade of $p$ ring resonators. Each ring resonator has a FSR of $N \Delta \omega / 2 p$, depending on the FFT stage. The cascade of ring resonators is followed by a ring resonator based bandpass filter (adddrop configuration) to suppress crosstalk and improve the filter performance. Fig. 1.a shows the design of the OOFDM demultiplexing system based on ring resonators for $N=4$ ( 4 subcarriers). The system is composed of the cascade of two ring resonators acting as a notch filter (only the output through port is used) and an additional bandpass filter (the drop port is used). The principle operation of the OOFDM demultiplexer is as follows. The OFDM signal is launched to the first ring resonator (RR1) through the input port. By properly adjusting the resonances position and the FSR for RR1, the OFDM subchannels adjacent to the desired subcarrier can be filtered out at its through port. Similar to RR1, the second ring resonator (RR2) extracts the further adjacent subchannels. Finally the desired OFDM subchannel is 
obtained at the drop port of the last ring resonator (RR3). Fig. 1.b shows the intensity transfer function of RR1 $\left(F_{S}\right)$, RR2 and the overall OOFDM demultiplexer. The phase response for the OOFDM demultiplexer based on ring resonators is also depicted.
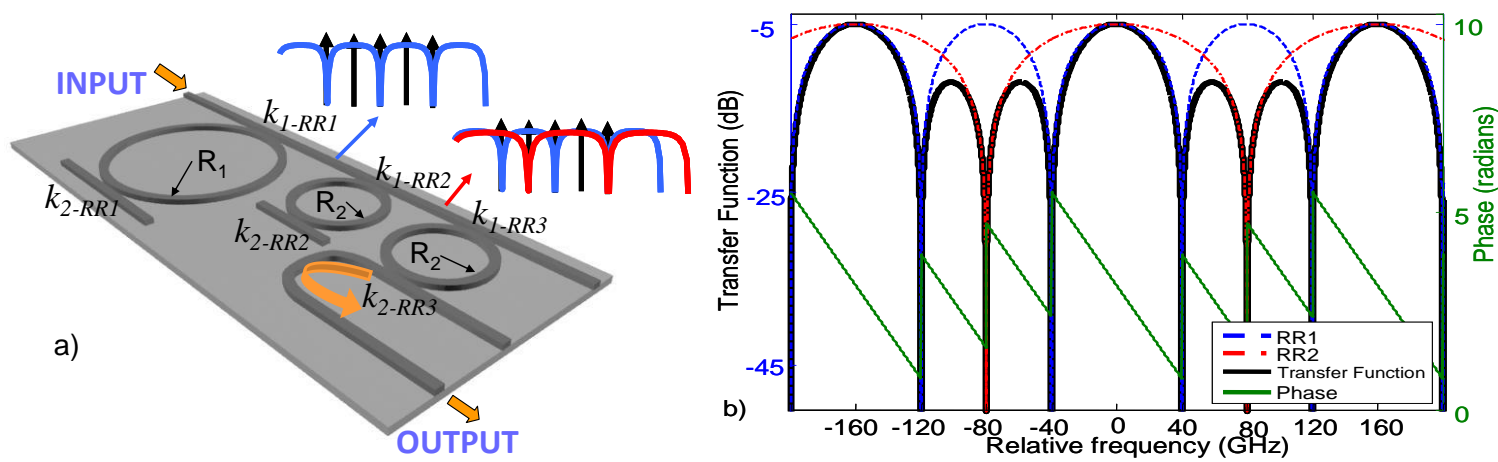

Figure 1. (a) Design of the OOFDM demultiplexing system based on cascaded ring resonators for $\mathrm{N}=4$ (4 subcarriers); (b) Intensity transfer function of RR1 (blue), RR2 (red), and the overall ring resonator based OOFDM demultiplexer for $\mathrm{N}=4$ (black). Green line represents the phase response of the OOFDM demultiplexer.

The simulation parameters for the ring resonators used for optical demultiplexing of a 4x40Gb/s OFDM signal are summarized in Table 1.

Table 1. Simulation parameters for the OFDM demultiplexer based on ring resonators.

\begin{tabular}{|l|c|c|c|}
\cline { 2 - 4 } \multicolumn{1}{c|}{} & Ring Resonator 1 (RR1) & Ring Resonator 2 (RR2) & Ring Resonator 3 (RR3) \\
\hline Radius & $132.77 \mu \mathrm{m}$ & $66.38 \mu \mathrm{m}$ & $66.38 \mu \mathrm{m}$ \\
\hline Coupling Ratios, $k_{l}$ & 0.7 & 0.65 & 0.6 \\
\hline Coupling Ratios, $k_{2}$ & 0.65 & 0.6 & 0.6 \\
\hline FSR & $80 \mathrm{GHz}$ & $160 \mathrm{GHz}$ & $160 \mathrm{GHz}$ \\
\hline$n_{e f f}$ & 2.8 & 2.8 & 2.8 \\
\hline$n_{g}$ & 4.5 & 4.5 & 4.5 \\
\hline Ring loss & $10 \mathrm{~dB} / \mathrm{cm}$ & $10 \mathrm{~dB} / \mathrm{cm}$ & $10 \mathrm{~dB} / \mathrm{cm}$ \\
\hline
\end{tabular}

Given the complementary behavior between the through and drop ports of a ring resonator, a simplification of the OOFDM demultiplexer can be obtained for performing simultaneous serial-to-parallel $(\mathrm{S} / \mathrm{P})$ conversion and demultiplexing of all subcarriers within an OFDM signal. By re-ordering the ring resonators, the number of elements required for demultiplexing an OFDM signal is reduced. The first stage of ring resonators (RR1-a, RR1-b) performs a filtering to separate data 1 and data 3 from data 2 and data 4 $(\mathrm{FSR}=80 \mathrm{GHz})$. In next stage, the second ring resonator with 160-GHz FSR (RR2-c, RR2-d) performs a filtering to select one OFDM subcarrier so that one OFDM subchannel appears at the through port and the other appears at the drop port. Final step (RR2-e, RR2-f, RR2-g, RR2-h) performs a filtering to improve the signal performance and each OFDM subcarrier is obtained in a specific output port of the device. Fig. 2.a shows the design of the simultaneous OFDM demultiplexing system and Fig. 2.b depicts the transfer function of each output port (data 1 , data 2 , data 3 and data 4).
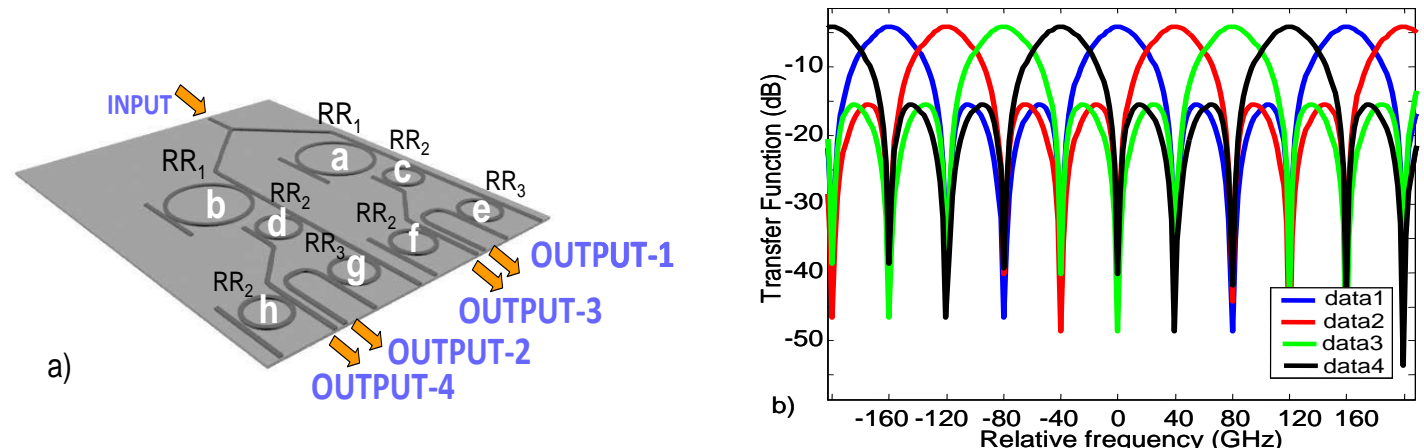

Figure 2. (a) Design of the simultaneous OOFDM demultiplexing system; (b) Intensity transfer function of each output port.

\section{Simulation of the OOFDM system using ring resonators as OOFDM demultiplexer}

To verify the feasibility of the OOFDM demultiplexing operation using cascaded silicon-based photonic ring resonators, simulations for the OOFDM system have been carried out by using VPI Photonics Inc. software. In Fig. 3 we show the schematic of the simulation set up used for the transmission of $160-\mathrm{Gb} / \mathrm{s}$ OFDM signals. The OFDM transmitter is similar to that shown in [4]. It employs a 40-GHz spaced optical frequency comb to generate 4 OFDM subcarriers (see Fig. 3.a). The comb is then filtered and each optical 
subcarrier is divided, individually modulated with an intensity modulator and finally coupled to create an OFDM signal (see Fig. 3.b). The bit rate of each subcarrier equals the optical subcarrier spacing in creating the OFDM signal. Indeed, each subcarrier is modulated by $40-\mathrm{Gb} / \mathrm{s}$ NRZ OOK data, obtaining an OFDM signal of $160 \mathrm{~Gb} / \mathrm{s}(4 \times 40 \mathrm{~Gb} / \mathrm{s})$. After forming the OFDM signal, a significant overlap of the channels spectra is observed and thus it can no longer be demultiplexed by standard bandpass optical filters without a high power penalty. At the receiver, simultaneous OOFDM demultiplexing is performed by using the ring resonator-based scheme shown in Fig. 2.a. The optically-demultiplexed OFDM subchannels at different subcarriers are finally photodetected after optical sampling, which is performed by using electro-absorption modulators (EAMs). A clearly opened eye pattern is observed at the output of the EAM, confirming that the RR-based scheme can be used for OOFDM demultiplexing (Figs. 3.e-h). To evaluate the performance of the all-optical RR-based OOFDM demultiplexer, bit error rate (BER) for each subcarrier has been measured, showing error-free operation, as shown in Fig. 4.

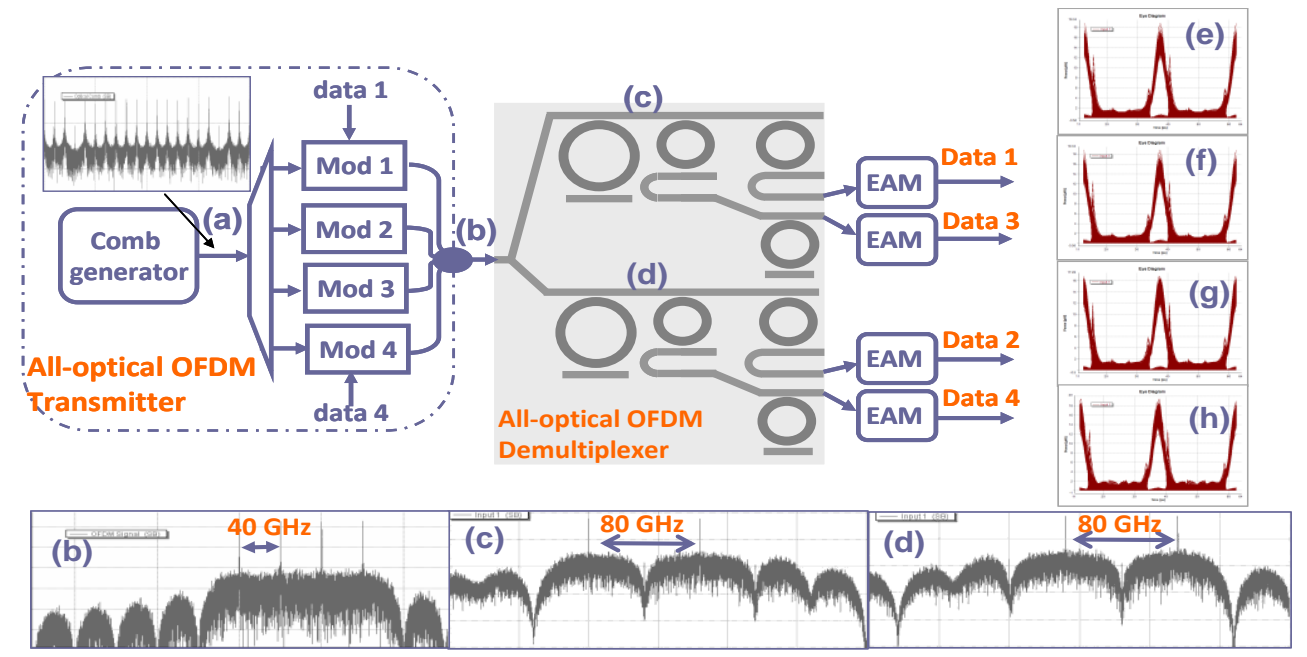

Figure 3. Set up of the OFDM system. (a) Output of the comb generator. (b) Four subcarriers of the comb source are filtered and modulated individually before being combined to form the $160-\mathrm{Gb} / \mathrm{s}$ OFDM signal. At the receiver, the subchannels are demultiplexed using the RR-based OOFDM demultiplexer. (c-d) Adjacent OFDM subchannels to the desired subcarrier can be filtered-out after the first RR (RR1). (e-h) Eye diagram of each OFDM subchannel.

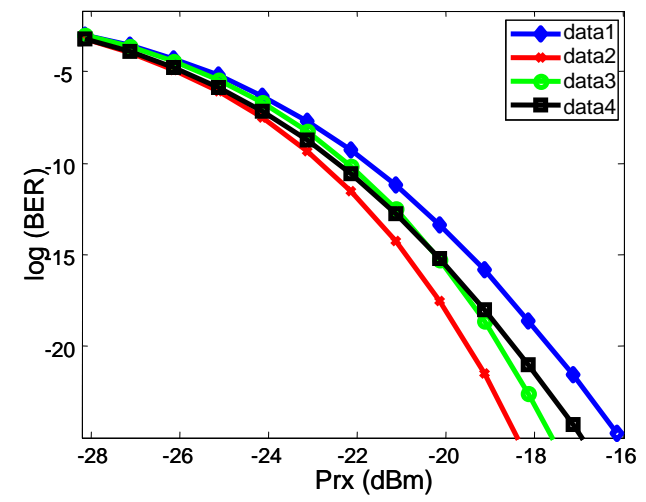

Figure 4. BER performance of each OFDM subchannel (data1, data2, data3, data4).

\section{Conclusion}

We propose a novel all-optical OFDM demultiplexer consisting of a cascade of silicon-based photonic ring resonators. The proposed OOFDM demultiplexer can be easily achieved using current fabrication technologies, thus providing a compact and cost-effective solution. A RR-based OFDM demultiplexing system for $4 \times 40-\mathrm{Gb} / \mathrm{s}$ OFDM signals is validated, showing excellent BER performance with error-free operation.

\section{References}

[1] J. Armstrong, “OFDM for Optical Communications”, IEEE/OSA J. Lightw. Technol., 27, 189-204 (2009).

[2] H. Sanjoh, E. Yamada, and Y. Yoshikuni, "Optical orthogonal frequency division multiplexing using frequency/time domain filtering for high spectral efficiency up to $1 \mathrm{bit} / \mathrm{s} / \mathrm{Hz}$ ”, in Proc. Of Conference on Optical Communication, OFC, Anaheim, CA, 2002, paper ThD1, 401-402 (2002).

[3] K. Takiguchi, M. Oguma, H. Takahashi, and A. Mori, "PLC-based eight-channel OFDM demultiplexer and its demonstration with 160 Gbit/s signal reception", in Proc. Of Conference on Optical Communication, OFC, San Diego, CA, 2010, paper OThB4 (2010). [4] D. Hillerhuss, M. Winter, M. Teschke, A. Marculescu, J. Li, G. Sigurdsson,K. Worms, S. Ben Ezra, N. Narkiss, W. Freude, and J. Leuthold, "Simple all-optical FFT scheme enabling Tbit/s real-time signal processing”, Opt. Express 18, 9324- 9340 (2010). 